\title{
microRNA-10b promotes the migration of mouse bone marrow-derived mesenchymal stem cells and downregulates the expression of E-cadherin
}

\author{
FENXI ZHANG ${ }^{1,2}$, SUHUA JING ${ }^{3}$, TONGMING REN ${ }^{1}$ and JUNTANG LIN ${ }^{2}$ \\ ${ }^{1}$ Department of Anatomy, Sanquan College, Xinxiang Medical University; \\ ${ }^{2}$ Stem Cell and Biotheraphy Technology Research Center, College of Life Science and Technology, Xinxiang Medical University; \\ ${ }^{3}$ Rehabilitation Center, The Third Hospital of Xinxiang Medical University, Xinxiang, Henan 453003, P.R. China
}

Received March 3, 2013; Accepted July 25, 2013

DOI: $10.3892 / \mathrm{mmr} .2013 .1615$

\begin{abstract}
The ability of mesenchymal stem cells (MSCs) to migrate is an important determinant of the efficiency of MSC transplant therapy. MicroRNA-10b (miR-10b) has been positively involved in the migration of a number of tumor cells lineages. To date, it remains unknown whether miR-10b affects the migration of MSCs. In the current study, the effect of miR-10b on the migration of mouse bone marrowderived MSCs (bmMSCs) was investigated. Third-passage bmMSCs were transfected with miR-10b mimic and negative control precursor miRNA using Lipofectamine ${ }^{\mathrm{TM}} 2000$. miR-10b and E-cadherin expression and bmMSC migration were determined. The present results showed that primary bmMSCs exhibit a spindled or triangular morphology and that third-passage bmMSCs present a typical fibroblast-like morphology, exhibiting CD90-positive and CD45-negative expression. Compared with the transfection of negative control miRNA, transfection of miR-10b mimic markedly upregulated miR-10b expression in bmMSCs, increased their migration and downregulated E-cadherin expression. The current observations indicate that the upregulation of miR-10b increases bmMSC migration ability, which may be involved in the downregulation of E-cadherin.
\end{abstract}

\section{Introduction}

Mesenchymal stem cells (MSCs) are multipotent cells that may differentiate into a variety of cell lineages, including osteocytes, adipocytes, chondrocytes, endothelial cells, cardiomyocytes and neurons, when exposed to appropriate conditions (1,2). Bone marrow-derived MSCs (bmMSCs) are

Correspondence to: Dr Fenxi Zhang, Department of Anatomy, Zhengxue Building, Sanquan College, Xinxiang Medical University, Jinshui East Road, Xinxiang, Henan 453003, P.R. China

E-mail: fxzhang0824@gmail.com

Key words: bone marrow-derived mesenchymal stem cells, microRNA-10b, cell migration, E-cadherin expression a commonly used source of stem cells. To date, bmMSCs have been widely applied in tissue engineering. The migration capability of bmMSCs is an important determinant of the efficiency of bmMSC-based transplant therapy. A previous study showed that $\sim 1.5 \%$ of injected stem cells reached the injured tissue following intracoronary injection for $2 \mathrm{~h}$ (3). However, the low homing rate of bmMSCs severely limits their clinical uses.

MicroRNAs (miRs) are endogenous, small, noncoding RNAs in eukaryotic cells (4). miRs are post-transcriptional regulators that negatively regulate gene expression by binding to the target mRNA for degradation and translational repression (4). At present, $>1000 \mathrm{miRs}$ have been identified in the human and mouse genomes, a number of which have been found to be involved in cell migration $(4,5)$. It has been reported that miRs, including miR-let-7a, -16, -30a, -34a, -107, $-125 b,-200 c,-203,-218,-424$ and -488 , inhibit the migration of specific tumor cells and other normal cell lineages (5-14). However, other miRs, including miR-10b, -20, -21 and -144, have been reported to promote cell migration (15-18).

To date, the effects of miR-10b on the migration and invasion of tumor cells have been well studied $(15,19,20)$. However, little is known about the function of miR-10b in the migration of bmMSCs. In the present study, the role of miR-10b in bmMSC migration and E-cadherin expression was investigated.

\section{Materials and methods}

Isolation and culture of bmMSCs. bmMSCs were isolated and cultured as previously described (21). In brief, bmMSCs were isolated from bone marrow, which was harvested from mouse tibia and femur, plated into 100-mm Petri dishes and cultured in DMEM (Invitrogen Life Technologies, Carlsbad, CA, USA) supplemented with $15 \%$ fetal bovine serum (Thermo Scientific HyClone, West Palm Beach, FL, USA), 2 mM L-glutamine (Sigma-Aldrich, St. Louis, MO, USA), $100 \mathrm{U} / \mathrm{ml}$ penicillin (Sigma-Aldrich) and $100 \mathrm{~g} / \mathrm{ml}$ streptomycin (Sigma-Aldrich) for $3 \mathrm{~h}$. The non-adherent cells were removed and the medium was replaced with fresh medium. A purified population of bmMSCs was obtained following 3 weeks of culture. The study was approved by the Ethics Committee of Xinxiang Medical University (Xinxiang, China) 

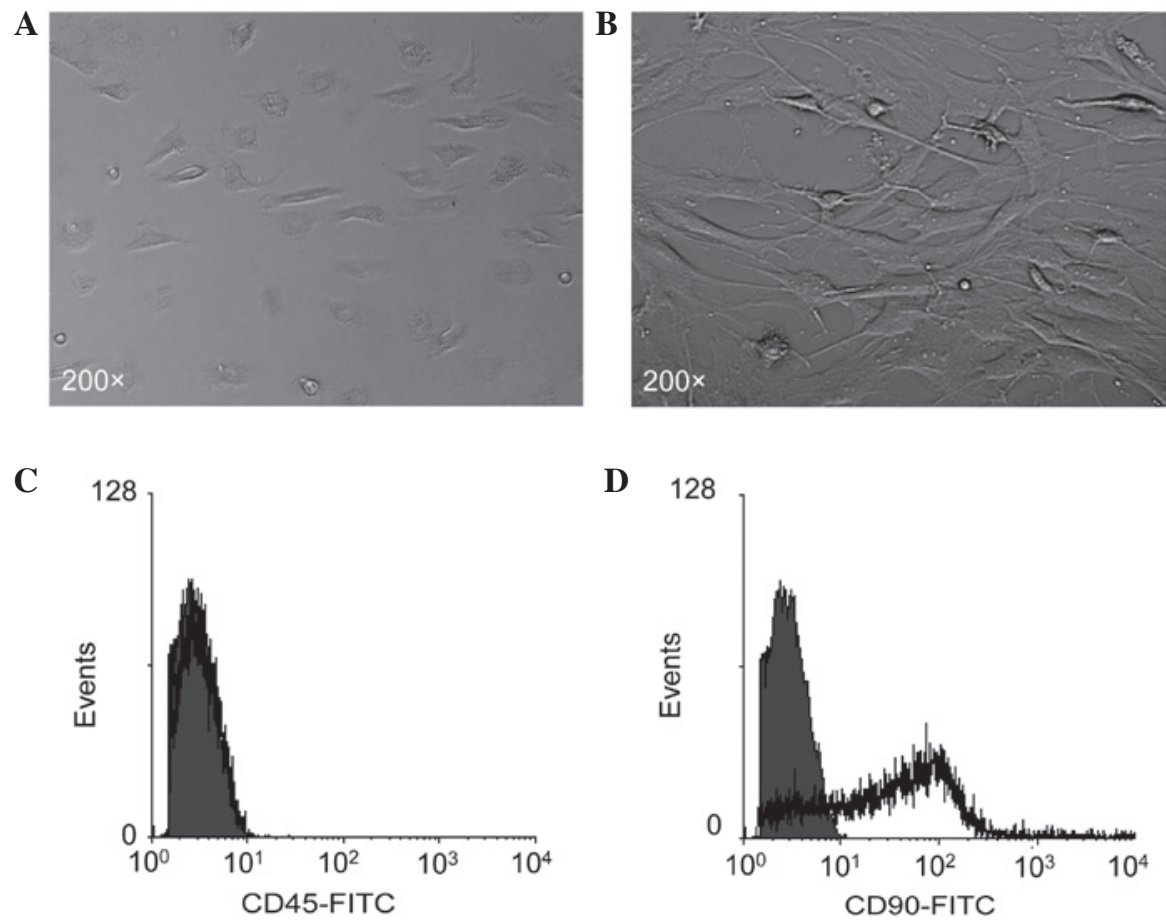

Figure 1. Morphology and identification of bmMSCs. (A) The morphology of primary of bmMSCs. (B) The morphology of the third-passage bmMSCs. (C) Flow cytometry assay revealed the expression of CD45 in bmMSCs. (D) Flow cytometry assay revealed the expression of CD90 in bmMSCs. bmMSCs, bone marrow-derived mesencymal stem cells.

Flow cytometry assay. bmMSCs were harvested, washed with phosphate-buffered saline (PBS) and incubated with CD45-FITC and CD90-FITC antibodies (Abcam, Cambridge, MA, USA) at $4^{\circ} \mathrm{C}$ for $1 \mathrm{~h}$. Cells were washed and resuspended in $400 \mathrm{ml}$ PBS and analyzed by flow cytometry (Becton-Dickinson, Franklin Lakes, NJ, USA). The flow cytometer collected $\sim 10,000$ cells.

Transfection of miR-10 mimic. bmMSCs were plated into 6-well plates. When cells reached $60-70 \%$ confluency, they were transfected with miR-10b mimic and negative control precursor miRNA using Lipofectamine ${ }^{\mathrm{TM}} 2000$ in Opti-MEM medium according to the instructions of the Lipofectamine LTX kit (Invitrogen Life Technologies, Carlsbad, CA, USA). The medium was replaced following $4 \mathrm{~h}$ of transfection. After $24 \mathrm{~h}$ of transfection, the cells were used in subsequent experiments.

Quantitative polymerase chain reaction ( $q P C R$ ) for $m i R-10 b$. bmMSCs transfected with miR-10b mimic or negative control miRNA were washed with PBS and total RNA was extracted using TRIzol reagent (Promega Corporation, Madison, WI, USA). miRNAs were purified using an miRNAeasy kit (Applied Biosystems, Carlsbad, CA, USA) and cDNA was synthesized using a microRNA reverse transcription kit (Applied Biosystems) according to the manufacturer's instructions. qPCR was performed using an Applied Biosystems 7500 Real-Time PCR System (Applied Biosystems). The miR-10b primers and U6 housekeeping primer were obtained from Abcam.

Transwell migration assay. The migration of bmMSCs was measured using Corning Costar transwell plates (Corning Inc.,
Corning, NY, USA) with $8-\mu \mathrm{m}$ pore filters, as previously described by Kim et al (22). In brief, bmMSCs $\left(1 \times 10^{5}\right)$ were plated in the upper inserts of the transwell chamber. Following $6 \mathrm{~h}$ of transmigration, the migrated bmMSCs on the lower side of the filter were fixed with $4 \%$ paraformaldehyde and stained with crystal violet and viewed by an inverted microscope (Olympus, Tokyo, Japan).

Immunofluorescence assay. Immunofluorescence staining was performed using rabbit anti-mouse E-cadherin antibody (Santa Cruz Biotechnology, Inc., Santa Cruz, CA, USA), as previously described (23). In brief, bmMSCs were cultured on $10-\mathrm{mm}$ round coverslips and stained using standard methods. Cells were mounted on slides using ProlongH Gold antifade reagent (Life Technologies Corporation, Carlsbad, CA, USA) and imaged by fluorescence microscopy (Olympus).

$R T-P C R$. Total RNA was isolated from bmMSCs using RNeasy mini kits (Invitrogen Life Technologies) according to the manufacturer's instructions. RNA $(1 \mu \mathrm{g})$ was applied to synthesize cDNA using the SuperScript II First Strand DNA Synthesis kit (Invitrogen Life Technologies). RT-PCR was performed using a $20-\mu 1$ reaction volume containing $100 \mathrm{ng}$ cDNA, $10 \mu 1$ 2X PCR mixture and $0.3 \mu \mathrm{M}$ primers. The products were separated by $1.5 \%$ agarose gel electrophoresis and visualized by ethidium bromide on a UV transilluminator (Bio-Rad, Hercules, CA, USA). The primers used were as follows: E-cadherin forward, 5'-CCTGTCAACCCAAGCAC-3' and reverse, 5'-ATTTCCTGACCCACACCAAA-3'; and $\beta$-actin forward, 5'-TTCTTTGCAGCTCCTTCGTTGCCG-3' and reverse, 5'-TGGATGGCTACGTACATGGCTGGG-3'. 
A

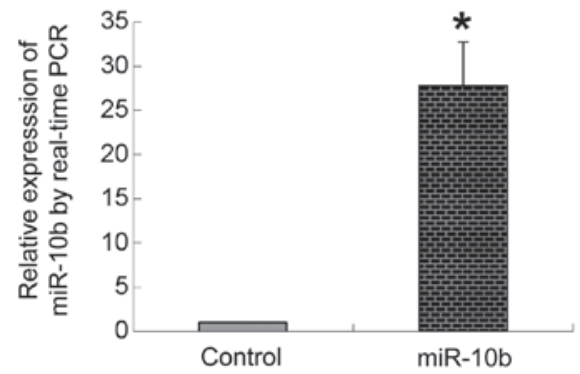

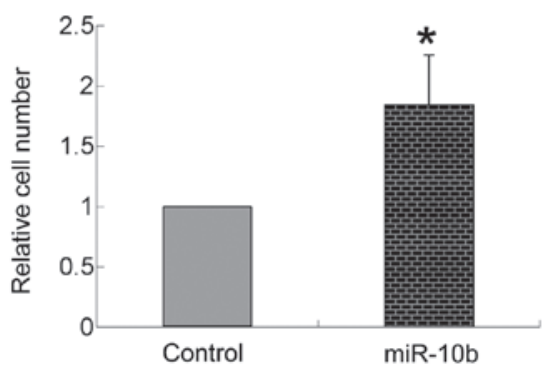

Figure 2. Expression of microRNA-10b (miR-10b) and cell migration following transfection of miR-10b mimic. (A) qPCR found miR-10b expression in bmMSCs transfected with negative control miRNA or miR-10b mimic. (B) Numbers of bmMSCs transmitted to the lower side of the well chamber following transfection of negative control miRNA or miR-10b mimic. Bar graphs represent mean $\pm \mathrm{SD}$ ( $\mathrm{n}=4$ /group). ${ }^{*} \mathrm{P}<0.05$ vs. negative control transfection. bmMSCs, bone marrow-derived mesencymal stem cells; qPCR, quantitative polymerase chain reaction.

A

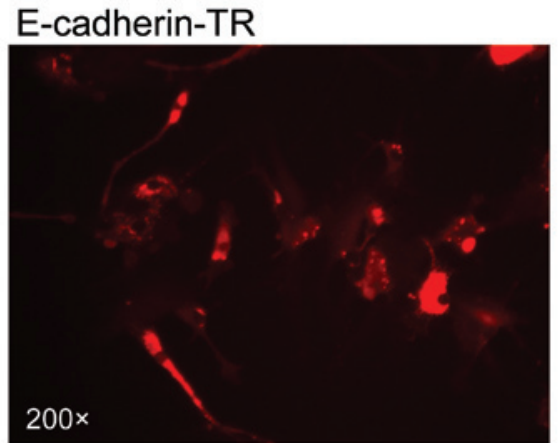

B

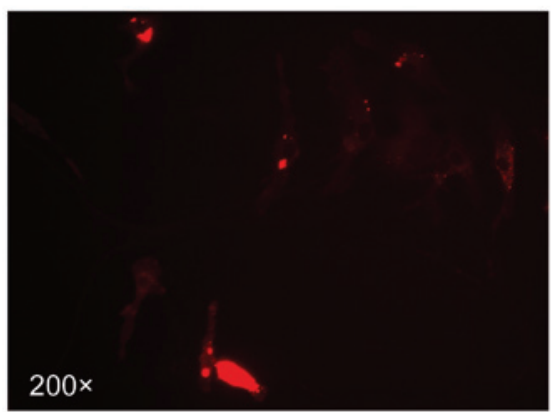

C
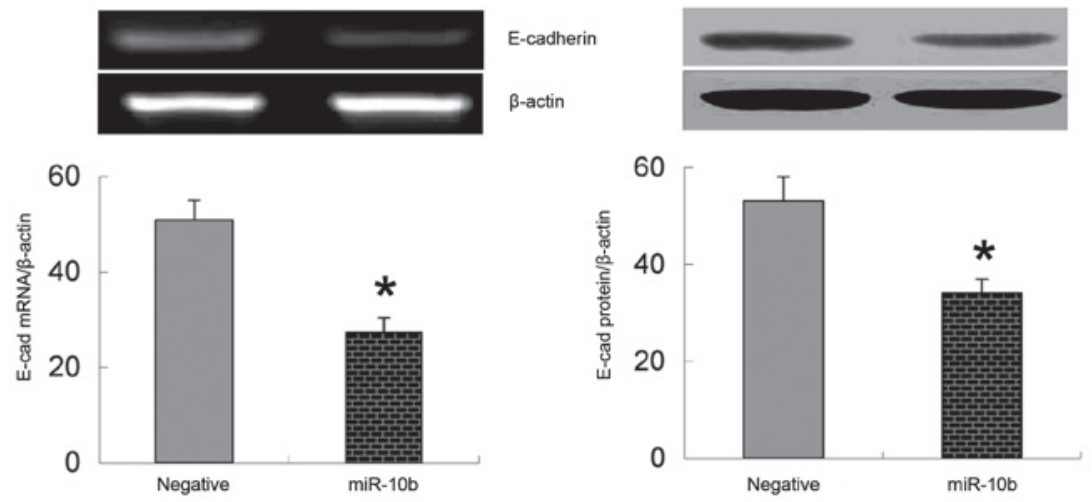

Figure 3. Expression of E-cadherin following transfection of microRNA-10b (miR-10b) mimic. (A) Immunofluorescence staining showed E-cadherin expression on the surface of bmMSCs transfected with negative control miRNA. (B) Immunofluorescence staining revealed E-cadherin expression on the surface of bmMSCs transfected with miR-10b mimic. (C) RT-PCR and western blot analysis identified mRNA and protein expression of E-cadherin in bmMSCs following transfection of negative control miRNA or miR-10b mimic. Bar graphs represent mean $\pm \mathrm{SD}$ ( $\mathrm{n}=4 / \mathrm{group}$ ). * $\mathrm{P}<0.05$ vs. negative control transfection. bmMSCs, bone marrow-derived mesencymal stem cells; RT-PCR, reverse-transcription polymerase chain reaction.

Western blotting. Proteins were extracted from bmMSCs and separated by $12 \%$ SDS-PAGE. Following electrophoresis, proteins were transferred to PVDF membranes. The membranes were blocked with 5\% non-fat milk in TBS-T and incubated with rabbit anti-mouse E-cadherin antibody at $4^{\circ} \mathrm{C}$ overnight. Blots were incubated with HRP-conjugated duck anti-rabbit secondary antibody (Santa Cruz Biotechnology, Inc.) for $1 \mathrm{~h}$ at room temperature. The immunoreactive bands were visualized by enhanced chemiluminescence.

Statistical analysis. Statistical analysis was performed with SPSS 11.5 software (SPSS Inc., Chicago, IL, USA). Data are presented as the mean \pm SD. Univariate comparison of means was evaluated using the Student's t-test. $\mathrm{P}<0.05$ was considered to indicate a statistically significant difference.

\section{Results}

Morphology of bmMSCs and expression of MSC markers. Consistent with previous studies (21), primary bmMSCs showed a spindle-shaped or triangular morphology (Fig. 1A) and the passaged (the third passage) bmMSCs exhibited a typical fibroblast-like morphology (Fig. 1B). Flow cytometry revealed that bmMSCs negatively expressed the leukocyte 
antigen molecule CD45 (Fig. 1C), but positively expressed the MSC marker molecule CD90 (Fig. 1D).

miR-10b expression following transfection. As shown in Fig. 2A, compared with the transfection of negative control precursor miRNA, transfection of miR-10b mimic markedly increased the expression of miR-10b in bmMSCs $(\mathrm{P}<0.05)$.

Overexpression of miR-10b promotes migration of bmMSCs. A transwell system was used to measure the transmigration ability of bmMSCs. As shown in Fig. 2B, compared with the transfection of negative control miRNA, transfection of the miR-10b mimic significantly increased the number of bmMSCs transmitted to the lower side of the well filters $(\mathrm{P}<0.05)$

Overexpression of miR-10b decreases expression of E-cadherin. It is known that E-cadherin is an important regulator of cell migration (24). Loss of cell surface E-cadherin suppresses cell adhesion and promotes cell migration (24). The present immunofluorescence data show that overexpression of miR-10b significantly decreases E-cadherin expression on the surface of bmMSCs (Fig. 3A and B). These observations were further confirmed by RT-PCR and western blot analysis, which indicate that mRNA and protein expression of E-cadherin are significantly downregulated in bmMSCs transfected with miR-10b mimic compared with those transfected with negative control miRNA ( $\mathrm{P}<0.05$; Fig. 3C).

\section{Discussion}

In the present study, upregulation of miR-10b was shown to promote the migration of bmMSCs in vitro for the first time. In addition, overexpression of miR-10b was observed to markedly decrease the expression of E-cadherin, a critical regulator of cell migration. These observations indicate that miR-10b positively regulates bmMSC migration, which may depend on its role in regulating E-cadherin expression.

miRs are small, noncoding RNA molecules that participate in multiple pathophysiological processes, including cell differentiation, migration, proliferation, apoptosis and inflammation (25). miR-10b is the a well-studied member of the miR family in cell metastasis. It has been shown that upregulation of miR-10b facilitates migration of several types of tumor cell lineages (19,20). For example, Tian et al (15) reported that overexpression of miR-10b increases the metastases of KYSE140 cells. Guessous et al (26) observed that miR-10b expression is increased in human glioblastoma tissues and glioblastoma stem cells, and inhibition of miR-10b markedly reduces the invasion and migration of glioblastoma stem cells. In the present study, overexpression of miR-10b was observed to significantly increase the migration of bmMSCs in the transwell assay.

E-cadherin is a transmembrane cell adhesion molecule that is important for multiple physiological processes, including cell migration, morphology and polarity (27). Downregulation of E-cadherin has been observed to decrease cell-cell adhesion and increase cell migration (28). In the present study, overexpression of miR-10b was observed to significantly decrease the expression of E-cadherin. miR-10b-mediated bmMSC migration is hypothesized to be involved in the downregulation of E-cadherin.

In summary, the current study supports the hypothesis that miR-10b promotes the migration of bmMSCs in vitro. The present observations indicate that the upregulation of $\mathrm{miR}-10 \mathrm{~b}$ expression may be a viable approach to increase the migration capacity of bmMSCs in transplantation therapy and an alternative to improve the therapeutic efficiency of transplantation.

\section{References}

1. Tokaer-Keskin Z, Akar AR, Ayaloglu-Butun F, et al: Timing of induction of cardiomyocyte differentiation for in vitro cultured mesenchymal stem cells: a perspective for emergencies. Can J Physiol Pharmacol 87: 143-150, 2009.

2. Oswad J, Boxberger S, Jørgensen B, et al: Mesenchymal stem cells can be differentiated into endothelial cells in vitro. Stem Cells 22: 377-384, 2004

3. Hu X, Wei L, Taylor TM, et al: Hypoxic preconditioning enhances bone marrow mesenchymal stem cell migration via Kv2.1 channel and FAK activation. Am J Physiol Cell Physiol 301: C362-C372, 2011.

4. Li C, Feng Y, Coukos G and Zhang L: Therapeutic microRNA strategies in human cancer. AAPS J 11: 747-757, 2009.

5. Kim SJ, Shin JY, Lee KD, et al: MicroRNA let-7a suppresses breast cancer cell migration and invasion through downregulation of C-C chemokine receptor type 7. Breast Cancer Res 14: R14, 2012.

6. Cheng CW, Wang HW, Chang CW, et al: MicroRNA-30a inhibits cell migration and invasion by downregulating vimentin expression and is a potential prognostic marker in breast cancer. Breast Cancer Res Treat 134: 1081-1093, 2012.

7. Yan K, Gao J, Yang T, et al: MicroRNA-34a inhibits the proliferation and metastasis of osteosarcoma cells both in vitro and in vivo. PLoS One 7: e33778, 2012.

8. Chen J, Chen XR, Zhang R, et al: MicroRNA-107 inhibits glioma cell migration and invasion by modulating Notch 2 expression. J Neurooncol 112: 59-66, 2013.

9. Wu D, Ding J, Wang L, et al: microRNA-125b inhibits cell migration and invasion by targeting matrix metallopeptidase 13 in bladder cancer. Oncol Lett 5: 829-834, 2013.

10. Jurmeister S, Baumann M, Balweierz A, et al: MicroRNA-200C represses migration and invasion of breast cancer cells by targeting actin-regulatory proteins FHOD1 and PPM1F. Mol Cell Biol 32: 633-651, 2012.

11. Takeshita N, Mori M, Kano M, et al: miR-203 inhibits the migration and invasion of esophageal squamous cell carcinoma by regulating LASP1. Int J Oncol 41: 1653-1661, 2012.

12. Kinoshita T, Hanazawa T, Nohata N, et al: Tumor suppressive microRNA-218 inhibits cell migration and invasion through targeting laminin-332 in head and neck squamous cell carcinoma. Oncotarget 3: 1386-1400, 2012.

13. Chamorro-Jorganes A, Araldi E, Penalva LO, et al: MicroRNA-16 and microRNA-424 regulate cell-autonomous angiogenic functions in endothelial cells via targeting vascular endothelial growth factor receptor-2 and fibroblast growth receptor-1. Arterioscler Thromb Vasc Biol 31: 2595-2606, 2011.

14. Song J, Kim D and Jin EJ: MicroRNA-488 suppresses cell migration through modulation of the focal adhesion activity during chondrogenic differentiation of chick limb mesenchymal cells. Cell Biol Int 35: 179-185, 2011.

15. Tian Y, Luo A, Cai Y, et al: MicroRNA-10b promotes migration and invasion through KLF4 in human esophageal cancer cell lines. J Biol Chem 285: 7986-7994, 2010.

16. Fan X, Liu Y, Jiang J, et al: miR-20a promotes proliferation and invasion by targeting APP in human ovarian cancer cells. Acta Biochim Biophys Sin (Shanghai) 42: 318-324, 2010.

17. Madhyastha R, Madhyastha H, Nakajima Y, et al: MicroRNA signature in diabetic wound healing: promotive role of miR-21 in fibroblast migration. Int Wound J 9: 355-361, 2012.

18. Zhang LY, Ho-Fun Lee V, Wong AM, et al: MicroRNA-144 promotes cell proliferation, migration and invasion in nasoparyngeal carcinoma through repression PTEN. Carcinogenesis 34: 454-463, 2013.

19. Li QJ, Zhou L, Yang F, et al: MicroRNA-10b promotes migration and invasion through CADM1 in human hepatocellular carcinoma cells. Tumour Biol 33: 1455-1465, 2012. 
20. Preis M, Gardner TB, Gordon SR, et al: MicroRNA-10b expression correlates with response to neoadjuvant therapy and survival in pancreatic ductal adenocarcinoma. Clin Cancer Res 17: 5812-5821, 2011.

21. Zhang F, Wang C, Jing S, et al: Lectin-like oxidized LDL receptor-1 expresses in mouse bone marrow-derived mesenchymal stem cells and stimulates their proliferation. Exp Cell Res 319: 1054-1059, 2013.

22. Kim YS, Kwon JS, Hong MH, et al: Promigratory activity of oxytocin on umbilical cord blood-derived mesenchymal stem cells. Artif Organs 34: 453-461, 2010.

23. Spencer HL, Eastham AM, Merry CL, et al: E-cadherin inhibits cell surface localization of the pro-migratory $5 \mathrm{~T} 4$ oncofetal antigen in mouse embryonic stem cells. Mol Biol Cell 18: 2838-2851, 2007.

24. Li L, Hartley R, Reiss B, et al: E-cadherin plays an essential role in collective directional migration of large epithelial sheets. Cell Mol Life Sci 69: 2779-2789, 2012.
25. Xiao Y, Xu C, Guan J, et al: Discovering dysfunction of multiple microRNAs cooperation in disease by a conserved microRNA co-expression network. PLoS One 7: e32201, 2012.

26. Guessous F, Alvarado-Velez M, Marcinkiewicz L, et al: Oncogenic effects of miR-10b in glioblastoma stem cells. J Neurooncol 112: 153-163, 2013.

27. Jamora $C$ and Fuchs E: Intercellular adhesion, signaling and the cytoskeleton. Nat Cell Biol 4: E101-E108, 2002.

28. Vogelmann R, Nguyen-Tat MD, Giehi K, et al: TGFbetainduced downregulation of E-cadherin-based cell-cell adhesion depends on PI3-kinase and PTEN. J Cell Sci 118: 4901-4912, 2005. 